\title{
Adaptación del cuestionario de alianza de ayuda revisado versión paciente (HAQ-II-P) para la población de cordobeses
}

\author{
Adaptation of the revised helping alliance questionnaire patient version \\ (HAQ-II-P) for the population of cordobans
}

\author{
Hanne Conn \\ Leonardo Adrián Medrano \\ Luciana Moretti \\ Universidad Empresarial Siglo 21, Córdoba, Argentina
}

(Rec: 26 de enero de 2012 / Acept:11 de abril de 2013)

\begin{abstract}
Resumen
La Psicología Basada en la Evidencia resultaría incompleta si no indagara el rol de la relación terapéutica. No obstante la ausencia de instrumentos adaptados obstaculiza el desarrollo de estudios empíricos en el contexto local. Este trabajo tuvo por objetivo principal adaptar la versión para pacientes del Cuestionario de Alianza de Ayuda Revisado (HAq-II). A su vez, como objetivo adicional se efectuó una comparación de tres modelos teóricos de alianza terapéutica. De esta manera, luego de traducir los ítems y evaluar la equivalencia mediante un panel de expertos se administró el HAq-II a una muestra de 119 participantes que se encontraban en tratamiento psicoterapéutico breve. Los resultados del análisis factorial confirmatorio sugieren un modelo subyacente de dos dimensiones: vinculo positivo con el terapeuta $(\alpha=.70)$ y acuerdo con los objetivos y tareas $(\alpha=.71)$. Cabe destacar que dicho modelo integra coherentemente las teorías propuestas por Luborsky y Bordin.

Palabras Clave. Alianza Terapéutica, Análisis Factorial Confirmatorio, Psicología Basada en la Evidencia.
\end{abstract}

\begin{abstract}
Evidence-Based Psychology would be incomplete if the role of the therapeutic relationship was not investigated. However, the absence of adapted instruments impedes the development of empirical studies in the local context. The principal objective of this work was to adapt the patient version of the Revised Helping Alliance Questionnaire (HAq-II). As an additional objective a comparison of three theoretical models of therapeutic alliance was carried out. After translating the items and evaluating their equivalency via a panel of experts, the HAq-II was administered to a sample of 119 participants undergoing brief psychotherapy. The results of the confirmatory factorial analysis suggest an underlying model of two dimensions: positive bond with the therapist $(\alpha=.70)$ and goal and task agreement $(\alpha=.71)$. It should be noted that this model fits coherently with the theories proposed by Luborsky and Bordin.

Key Words. Therapeutic Alliance, Confirmatory Factorial Analysis, Evidence-Based Psychology.
\end{abstract}

\section{Introducción}

No es posible concebir la psicoterapia sin tener en cuenta el establecimiento de una relación interpersonal entre el cliente y el terapeuta (Corbella y Botella, 2003). En efecto independientemente del modelo teórico de base las diferentes tradiciones psicoterapéuticas acuerdan en mayor o menor medida en que el establecimiento de una relación positiva y de colaboración con el paciente resulta indispensable para alcanzar el éxito psicoterapéutico. Si bien los inicios del estudio de la relación terapéutica se inician con Freud, tanto el movimiento humanista como la vertiente cognitiva enfatizaron la importancia de esta relación. De esta manera Roger señaló que ser empático, congruente y aceptar incondicionalmente al cliente eran características imprescindibles que debía tener un terapeuta

Correspondencia: 15 Ardilaun Road, London, N5 2QR, Inglaterra. León 1955, Barrio Maipú Córdoba, Argentina. Cp: 5000. Email: hanne.conn@gmail. com leomedpsico@gmail.com, 1smoretti@gmail.com. 
para el establecimiento de una relación efectiva y curativa con el cliente. Asimismo autores cognitivos como Beck, Rush, Shaw y Emery (2005) sugieren la importancia de una relación basada en la confianza, el rapport y la colaboración, así como la necesidad de que el terapeuta desarrolle aceptación, empatía y autenticidad para fomentar la relación terapéutica.

Entre los componentes de esta relación, la alianza terapéutica es la que ha recibido más atención por parte de teóricos e investigadores (Gelso y Carter, 1994). Tradicionalmente la alianza ha sido definida sobre la base de tres elementos: a) acuerdo en las tareas, dado que el paciente debe considerar que las acciones y pensamientos que forman parte del proceso terapéutico son relevantes para su mejoría, b) vínculo positivo, debe existir confianza y aceptación mutua entre paciente y terapeuta; y c) acuerdo en los objetivos que se esperan alcanzar a través de la psicoterapia (Bordin, 1976). Más recientemente Luborsky (1994) conceptualizó la alianza de ayuda, de manera general, como la expresión de un vínculo positivo que el paciente tiene con su terapeuta, el cual está percibido por el paciente como una persona que proporciona ayuda y apoyo. Este autor considera la alianza como un fenómeno dinámico que se desarrolla a través del proceso terapéutico y describe dos tipos de alianza en función de la fase de la psicoterapia; la alianza de tipo uno representa una relación de ayuda que se da más que nada en el comienzo de la terapia, y que depende de que el paciente tenga una sensación de apoyo y de ayuda proporcionada por el terapeuta. Los seis signos definidos por Luborsky (1994) de la alianza de tipo uno son: 1) el paciente siente que el terapeuta es cálido y que proporciona apoyo; 2) el paciente cree que el terapeuta le ayuda; 3) el paciente se siente cambiado debido a la terapia, 4) el paciente siente un rapport con el terapeuta; 5) el paciente siente que el terapeuta le respeta y le valora; 6) el paciente expresa creencias acerca del valor del proceso terapéutico.

La alianza de tipo dos se da en fases posteriores, caracterizada por una relación de ayuda que consiste en una sensación de trabajo conjunto destinado a la superación de los obstáculos y el malestar del paciente (Luborsky, 1994). Los cuatro signos de esta alianza son: 1) el paciente experimenta la relación como un trabajo y esfuerzo conjunto; 2) el paciente comparte concepciones similares acerca del origen de los problemas con el terapeuta; 3) el paciente expresa la creencia de que es cada vez más capaz de cooperar con el terapeuta; y 4) el paciente demuestra capacidades similares a las del terapeuta en términos de poder utilizar herramientas para comprender.
En este punto es importante señalar que Corbella y Botella (2003) plantean que, a pesar de las diferencias en las teorizaciones de Luborsky (1994) y Bordin (1976), es posible encontrar una complementariedad entre ambas. La alianza de tipo uno de Luborsky (1994) se puede relacionar con el elemento del vínculo positivo de Bordin, y la alianza de tipo dos podría relacionarse con el acuerdo en las tareas y los objetivos en Bordin.

El interés por el estudio de la relación terapéutica se ha visto revitalizado en las últimas décadas a partir de los nuevos desarrollos en el movimiento de Psicología Basada en la Evidencia (Stevens y Méndez, 2007). En efecto en el año 1999, la División 29 de la APA (Psicoterapia) estableció una comisión para identificar, operacionalizar y diseminar información sobre la relación terapéutica con apoyo empírico, estudiando la asociación entre resultados y aspectos de la relación y la alianza terapéutica (Norcross, 2001, American Psychological Association, 2006).

Según Martin, Garske y Davis (2000) el incremento en la investigación acerca de la alianza se debe a la relación constante que se encuentra entre la calidad de la alianza y los resultados terapéuticos, una relación que se mantiene a pesar de las diferentes orientaciones teóricas y maneras disímiles de evaluar la alianza entre investigadores (Fenton, Cecero, Nich, Frankforter y Carroll, 2001; Horvath y Symonds, 1991; Luborsky, 1994; Martin, Garske y Davis, 2000; Summers y Barber, 2003). Ciertamente, Marmar, Gaston, Gallagher y Thompson (1989) postulan que la alianza predice de manera significativa los resultados de modalidades diferentes de psicoterapia y, a la vez, se ha encontrado que esta relación se mantiene en el tratamiento de problemas psicológicos variados (Fenton et al., 2001).

Otra razón por el incremento en el interés en la alianza es la inhabilidad de los investigadores para encontrar diferencias sistemáticas en la efectividad de las psicoterapias de las diferentes orientaciones teóricas (Martin, Garske y Davis, 2000). Dada la conclusión de que las psicoterapias son, por lo general, efectivas (Goodheart, Kazdin y Stenberg, 2006), muchos investigadores han empezado a buscar factores comunes a todas las terapias para así poder explicar los resultados terapéuticos. En efecto, Lambert (citado en Gómez, 2010) encontró que de los factores comunes de la psicoterapia, la alianza es uno de los principales dado que explica aproximadamente un $30 \%$ de la mejoría del paciente. En este sentido algunos investigadores como Safran y Muran (2003) sostienen que la calidad de la alianza es más importante que la modalidad de terapia utilizada en la predicción de los resultados terapéuticos. No obstante es importante señalar que una buena alianza no es curativa por 
sí misma, sino que es un factor que hace posible la aceptación y el seguimiento del trabajo terapéutico (Horvath y Greenberg, 1994).

De acuerdo con Corbella y Botella (2003), se han creado más de veinte instrumentos para medir la alianza, de los cuales los de mayor uso son el Cuestionario de Alianza de Ayuda Revisado (Revised Helping Alliance Questionnaire HAq-II) de Luborsky et al. (1996), el Inventario de Alianza de Trabajo (Working Alliance Inventory-WAI) de Horvath y Greenberg (1986) y las Escalas de Alianza Psicoterapéutica de California (California Psychotherapy Alliance Scales CALPAS) de Gaston (1991). Cada uno de estos instrumentos cuenta con una versión para terapeutas, otra para pacientes y una para un tercer observador.

En la actualidad, en Argentina no existen adaptaciones de dichos tests para su uso en el contexto local. En este sentido es importante señalar que la utilización de este tipo de cuestionarios en contextos culturales ajenos al contexto de creación del instrumento puede generar mediciones poco válidas y confiables debido a sesgos provenientes de las características de las muestras de estandarización, el idioma, la familiaridad con los ítems del test y la modalidad de aplicación. Por ello aplicar un instrumento en un contexto que no sea el de su origen sin efectuar los análisis psicométricos correspondientes constituye una práctica inadecuada (Fernández, 2008; Hambleton y Patsula, 1999; Muñiz y Hambleton, 1996).

En el presente estudio se optó por adaptar la versión para pacientes del Cuestionario de Alianza de Ayuda Revisado (HAq-II). Si bien Crits-Christoph, Gladis y Connolly (2002) señalan que las propiedades psicométricas de los tres instrumentos de medición anteriormente mencionados son buenas (la CALPAS, el WAI y el HAq-II demuestran buena consistencia interna, son representaciones válidas de la alianza como constructo, y se correlacionan altamente entre sí), además para uso en la clínica sería más provechoso utilizar el HAq-II, ya que es la escala más corta de las tres, y dado que el completar el cuestionario puede ser una actividad ansiógena para el paciente, la elección de la escala igualmente confiable y válida pero con el menor número de ítems podría ser preferible. Asimismo se optó por la versión para pacientes dado que hay evidencia de que las versiones para paciente predicen mejor los resultados de la terapia que las versiones para terapeuta (Horvath y Symonds, 1991).

Además de efectuar estudios tendientes a evaluar las propiedades psicométricas de la versión adaptada del HAqII-P en la población cordobesa, se plantea como objetivo adicional comparar el ajuste de tres modelos teóricos de alianza terapéutica, más concretamente se comparan el modelo original del HAq-II-P en el que subyacen dos dimensiones de la alianza terapéutica (alianza positiva y alianza negativa; Luborsky et al., 1996), el modelo reportado por Corbella y Botella (2003) donde se integran las teorías de Luborsky (1994) y Bordin (1976) diferenciando la alianza tipo uno (vínculo positivo) y la alianza tipo dos (acuerdo en los objetivos y las tareas), y, finalmente, un modelo de alianza terapéutica unifactorial donde los ítems del HAqII-P se agruparían en un único factor.

\section{Método}

\section{Participantes}

La muestra está compuesta por 119 participantes seleccionados a través de un muestro de tipo no probabilístico a propósito (León y Montero, 2003). Un 21\% de la muestra es masculino y $79 \%$ femenino. Para ser coherente con la muestra utilizada en el estudio psicométrico de la escala original, se fijó que los participantes debían tener entre 19 y 59 años, estar en tratamiento psicoterapéutico breve con un psicólogo o un psiquiatra, y encontrarse en la segunda sesión en adelante de su terapia.

\section{Instrumento}

Cuestionario de Alianza de Ayuda Revisado, Versión Paciente (Revised Helping Alliance Questionnaire, Patient Version-HAq-II-P: El cuestionario se creó a partir de la revisión del Cuestionario de Alianza de Ayuda (Helping Alliance Questionnaire-HAq) de Alexander y Luborsky (1986) (citado en Luborsky et al., 1996). Los cambios realizados al revisar el HAq eran necesarios por dos razones principales (Luborsky et al., 1996): primero, porque el cuestionario contenía ítems que explícitamente medían mejoría sintomática temprana, la cual, de acuerdo con las teorías de Luborsky (1994) y Bordin (1976), no es un componente de la alianza. La segunda razón es que todos los ítems del HAq estaban expresados de manera positiva. Entonces, los autores eliminaron los seis ítems que median mejoría temprano y agregaron unos catorce ítems, de los cuales cinco están expresados de manera negativa. Según Le Bloc'h, de Roten, Drapeau y Despland (2006), otra mejoría importante de la escala es que el HAq-II es más representativo de las dimensiones de la alianza que el HAq, en especial lo referente a los aspectos colaborativos entre paciente y terapeuta. A partir de estos cambios entonces se creó un cuestionario nuevo de 17 ítems el HAq-II (el mismo 
parte de un pool inicial de 19 ítems). Los ítems se agrupan en dos subescalas: "alianza terapéutica positiva" y "alianza terapéutica negativa", y son de tipo likert con escalas de respuesta de 6 puntos que van desde "muy en desacuerdo" hasta "muy de acuerdo". Le Bloc'h, de Roten, Drapeau y Despland (2006) recomiendan utilizar el HAq-II en lugar del HAq, ya que efectivamente el HAq-II se relaciona mejor con el constructo de alianza de acuerdo con las teorías de Bordin (1976) y Luborsky, (1994) y está menos influenciado por la sintomatología del paciente.

Luborsky et al. (1996) encontraron una consistencia interna (utilizando el alfa de Cronbach) de $.90, .90$ y .93 en las sesiones 2, 5 y 24 respectivamente, y una confiabilidad test-retest entre las sesiones 2 y 5 de $.78(p<.005)$. El estudio de la estructura interna del cuestionario dio por resultado 2 factores: el primer factor, "alianza terapéutica positiva" se constituyó por los ítems $1,2,3,5,6,7,9,10,12,13$, 15, 17 y 18 y explicó el $43.4 \%$ de la varianza. El segundo factor "alianza terapéutica negativa" estuvo compuesto por los ítems 4, 8, 16 y 19 y explicó un $10,6 \%$ de la varianza. En la segunda sesión la correlación entre los dos factores era $r=.48(p<.001)$, en la sesión $5 r=.60(p<.001)$ y en la sesión numero $24 r=.64(p<.001)$.

En cuanto a los estudios de validez externa, el instrumento demostró una adecuada validez convergente con otro instrumento de medición de alianza, las Escalas de California de Alianza Psicoterapéutica (California Psychotherapy Alliance Scales-CALPAS). En la sesión numero dos la correlación entre el HAq-II-P y la CALPAS era de $.59(p$ $<.001)$, en la sesión 5 era de $.68(p<.001)$ y en la sesión 24 era de $.69(p<.001)$.

Con respecto a la validez discriminante, se midieron las correlaciones entre la alianza y la edad, genero, estado civil y empleo de los participantes en las sesiones 2 y 5 y como se esperaba no se encontró correlación. También se midieron las correlaciones entre la alianza en las sesiones 2 y 5 y las medidas pretratamiento de disfunción psiquiátrica y uso de drogas: no se encontraron relaciones entre la escala y el nivel de funcionamiento psicológico, severidad psiquiátrica, uso de drogas o nivel de depresión de los pacientes al ingresar al tratamiento.

\section{Procedimiento}

Se realizó un estudio de tipo instrumental (Montero y León, 2007). En un primer momento un experto realizó la traducción de los ítems del HAq-II-P del inglés al castellano. Luego, tres expertos juzgaron la calidad de la traducción de los ítems en una escala de 1 a 10 y se efectuó el cálculo del coeficiente $\mathrm{V}$ de Aiken para estimar el grado de acuerdo de los jueces en relación a la calidad de la traducción. Posteriormente, se realizó un estudio piloto con cinco participantes con el fin de identificar si los reactivos traducidos requerían de ajustes adicionales, para lo cual se evaluaron aspectos como el lenguaje y vocabulario utilizado, la comprensión de las opciones de respuesta, la comprensión de los ítems, entre otros.

Una vez efectuado dichos estudios se administró el cuestionario a una muestra de 119 participantes con el previo consentimiento informado de los participantes y garantizando el anonimato de su participación. A partir de los datos recabados se realizó un análisis factorial confirmatorio utilizando el programa AMOS 17 para analizar la estructura factorial subyacente. A continuación se estimó la consistencia interna mediante el coeficiente alfa de Cronbach. Por último, se efectuó un estudio de comparación de grupos según el género de los participantes y el tipo de terapeuta (psicólogo o psiquiatra), dichos análisis se calcularon utilizando el paquete estadístico para ciencias sociales SPSS 17.

\section{Resultados}

\section{Fase 1: Traducción y juicio de la calidad de traducción}

Un investigador experto bilingüe (inglés como lengua nativa) realizó la traducción de los ítems de la escala original del inglés al castellano. Tal como sugiere la literatura se realizó una revisión de las traducciones efectuadas a través de un panel de expertos (Coffman, 2008). Este tipo de estudios provee información valiosa sobre las posibilidades de aplicabilidad a la población, la adecuación de la traducción y la equivalencia de los ítems traducidos. De esta manera tres expertos juzgaron la adecuada traducción de cada ítem, otorgando una valoración entre 1 y 10 en función de que los ítems en castellano reflejaran una adecuada traducción de los ítems originales en inglés. Posteriormente se efectuó el cálculo V de Aiken para estimar el acuerdo entre los jueces en relación a la calidad de la traducción de cada ítem. Para la estimación del coeficiente V y sus intervalos de confianza (para un nivel de confianza del 90\%) se utilizó el programa desarrollado por Soto y Segovia (2009). Como se puede apreciar en la tabla 1, todos los valores $\mathrm{V}$ obtenidos superan el valor recomendado de $V=.70$ y ninguno de los 
Tabla 1. Evaluación de la calidad de la traducción de los items (intervalo considerado para un 95\% de confianza).

\begin{tabular}{|c|c|c|c|}
\hline Ítems & $\mathrm{V}$ & Límite superior & Límite inferior \\
\hline 1. Siento que puedo depender del terapeuta & 0.97 & 0.82 & 0.99 \\
\hline 2. Siento que el terapeuta me entiende & 0.92 & 0.76 & 0.97 \\
\hline 3. Siento que el terapeuta quiere que yo logre mis metas & 1 & 0.87 & 1 \\
\hline 4. A veces desconfío del juicio del terapeuta & 1 & 0.87 & 1 \\
\hline 5. Siento que estoy trabajando con el terapeuta en un esfuerzo conjunto & 0.85 & 0.67 & 0.94 \\
\hline 6. Creo que ambos tenemos ideas similares acerca de la naturaleza de mi problema & 1 & 0.87 & 1 \\
\hline 7. Generalmente respeto las ideas que el terapeuta tiene de mí & 0.81 & 0.62 & 0.91 \\
\hline $\begin{array}{l}\text { 8. Los procedimientos utilizados en mi terapia no son muy apropiados para mis } \\
\text { necesidades }\end{array}$ & 0.92 & 0.76 & 0.97 \\
\hline 9. El terapeuta me agrada como persona & 1 & 0.87 & 1 \\
\hline $\begin{array}{l}\text { 10. En la mayoría de las sesiones el terapeuta y yo encontramos una forma de } \\
\text { trabajar juntos sobre mis problemas }\end{array}$ & 0.92 & 0.76 & 0.97 \\
\hline 11. Se ha formado una buena relación con mi terapeuta & 0.85 & 0.67 & 0.94 \\
\hline 12. El terapeuta parece tener experiencia en ayudar a la gente & 1 & 0.87 & 1 \\
\hline 13. El terapeuta y yo tenemos conversaciones significativas & 0.88 & 0.71 & 0.96 \\
\hline 14. A veces, el terapeuta y yo tenemos conversaciones no provechosas & 0.97 & 0.82 & 0.99 \\
\hline $\begin{array}{l}\text { 15. De vez en cuando, ambos hablamos sobre los mismos eventos importantes } \\
\text { en mi pasado }\end{array}$ & 0.81 & 0.62 & 0.91 \\
\hline 16. Creo que le agrado al terapeuta como persona & 0.85 & 0.67 & 0.94 \\
\hline 17. A veces el terapeuta parece distante & 1 & 0.87 & 1 \\
\hline
\end{tabular}

intervalos inferiores presenta valores por debajo del valor $V=.60$. En función de ello no se realizaron cambio a los ítems traducidos.

Posteriormente al juicio de calidad de la traducción se realizó un estudio piloto con cinco participantes con el fin de detectar deficiencias del cuestionario y de la planificación del trabajo de campo. Se encontró que no había problemas en cuanto a la administración del cuestionario, la comprensión de las opciones de respuesta ni la longitud del instrumento. El cuestionario resultó fácil y atractivo y no despertó respuestas ego defensivas. No obstante, sí hubo dificultad con la comprensión del vocabulario utilizados en los ítems 15 y 16, y como resultado se decidió cambar la palabra "intercambios" a "conversaciones".

\section{Fase 2: Análisis de estructura interna del HAq-II-P: comparación de modelos teóricos}

Tal como se especificó en la introducción del trabajo se planteó como objetivo adicional comparar el ajuste de tres modelos de alianza terapéutica. Tal como se presenta en la tabla 2, en el Modelo 1 los ítems se distribuyen en dos dimensiones subyacentes denominadas alianza terapéutica positiva y alianza terapéutica negativa, este modelo es el originalmente propuesto por Luborsky et al. (1996). Atendiendo a la integración comunicada por Corbella y Botella (2003) de las teorías de Luborsky (1994) y Bordin (1976) se planteó un Modelo 2 en donde los ítems se agrupan en dos dimensiones, el primer tipo de alianza refiere al vínculo positivo entre el terapeuta y el cliente, mientras que el segundo tipo de alianza refiere al acuerdo en los objetivos y tareas. En función del contenido de los ítems los mismos fueron ordenados en cada dimensión, de esta manera la primera dimensión quedó integrada por 6 reactivos ("Se ha formado una buena relación con mi terapeuta", por ejemplo) y la segunda dimensión también quedó integrada por 6 reactivos ("Creo que ambos tenemos ideas similares acerca de la naturaleza de mi problema", por ejemplo), en este modelo se descartan 5 ítems dado que no reflejaban teóricamente ninguna de las dos dimensiones propuestas. Finalmente se formuló un Modelo 3 en donde se plantea un modelo unidimensional de alianza terapéutica.

Antes de proceder con el análisis factorial confirmatorio se examinó mediante la normalidad univariada y multivariada 
Tabla 2. Distribución de los reactivos del HAq-II-P según el modelo teórico de base.

\begin{tabular}{|c|c|c|c|c|c|}
\hline & \multicolumn{2}{|c|}{ Modelo 1} & \multicolumn{2}{|c|}{ Modelo 2} & \multirow{2}{*}{$\frac{\text { Modelo } 3}{\text { Alianza terapéutica }}$} \\
\hline Ítems del HAq-II-P & Alianza positiva & Alianza negativa & Vínculo positivo & $\begin{array}{c}\text { Acuerdo en objetivos y } \\
\text { tareas }\end{array}$ & \\
\hline 1 & $\mathrm{x}$ & & & & $\mathrm{x}$ \\
\hline 2 & $\mathrm{x}$ & & $\mathrm{x}$ & & $\mathrm{x}$ \\
\hline 3 & $\mathrm{x}$ & & & $\mathrm{x}$ & $\mathrm{x}$ \\
\hline 4 & & $\mathrm{x}$ & & & x (i) \\
\hline 5 & $\mathrm{x}$ & & & $\mathrm{x}$ & $\mathrm{x}$ \\
\hline 6 & $\mathrm{x}$ & & & $\mathrm{x}$ & $\mathrm{x}$ \\
\hline 7 & $\mathrm{x}$ & & & & $\mathrm{x}$ \\
\hline 8 & & $\mathrm{x}$ & & x (i) & x (i) \\
\hline 9 & $\mathrm{x}$ & & $\mathrm{x}$ & & $\mathrm{x}$ \\
\hline 10 & $\mathrm{x}$ & & & $\mathrm{x}$ & $\mathrm{x}$ \\
\hline 11 & $\mathrm{x}$ & & $\mathrm{x}$ & & $\mathrm{x}$ \\
\hline 12 & $\mathrm{x}$ & & & & $\mathrm{x}$ \\
\hline 13 & $\mathrm{x}$ & & & & $\mathrm{x}$ \\
\hline 14 & & $\mathrm{x}$ & & x (i) & x (i) \\
\hline 15 & $\mathrm{x}$ & & $\mathrm{x}$ & & $\mathrm{x}$ \\
\hline 16 & $\mathrm{x}$ & & $\mathrm{x}$ & & $\mathrm{x}$ \\
\hline 17 & & $\mathrm{x}$ & x (i) & & x (i) \\
\hline
\end{tabular}

Nota(i). ítems en que se invirtió su puntaje.

de los ítems. Se observaron valores de asimetría y curtosis comprendidos entre los valores \pm 2 , los cuales resultan óptimos considerando los criterios propuestos por George y Mallery (2010). Asimismo el índice Mardia de normalidad multivariada presentó un valor de 45.25 encontrándose por debajo del valor crítico de 70 sugerido por Rodríguez Ayán y Ruiz (2008).

A continuación se procedió con la estimación del modelo utilizando el método de Máxima Probabilidad ya que es el método más utilizado, eficiente y no sesgado cuando las variables poseen una distribución normal (Byrne, 2001). Para evaluar el ajuste de los modelos se consideró el índice de chi cuadrado de bondad de ajuste $(\chi 2)$, el índice de bondad del ajuste (GFI), el índice de ajuste comparado (CFI) y el índice de la raíz cuadrada media del error de aproximación (RMSEA), tal como sugiere la literatura especializada (Byrne, 2001).

Tal como puede apreciarse en la tabla 3 el Modelo 2 es el que presente mejor ajuste al compararlo con el modelo 1 y 3. Cabe considerar también que el ajuste resulta aceptable considerando los criterios establecidos por Hu y Bentler (1995). Los coeficientes de regresión estandarizados obtenidos se presentan en la figura 1 .

\section{Fase 3: Análisis de consistencia interna y estudio de} contrastación de Grupos

Una vez delimitados los factores subyacentes del HAqII-P se procedió a calcular la consistencia interna de cada dimensión. Para la dimensión 1 "Alianza Positiva" se obtuvo un coeficiente $\alpha=.70$, mientras que para la dimensión 2 "Acuerdo con los Objetivos y Tareas" presentó un $\alpha=.71$. Los resultados son satisfactorios siguiendo los criterios propuestos por Oviedo y Campos-Arias (2005) donde valores $\alpha$ menores a .70 indican una pobre consistencia interna y no son aceptables, valores entre .70 y .90 indican una buena consistencia interna y valores superiores a .90 pueden indicar que hay duplicación de los elementos del constructo, y por ende los ítems redundantes que deben eliminarse. 
Tabla 3. Índices de ajustes de los modelos de ecuaciones estructurales.

\begin{tabular}{|c|c|c|c|c|c|c|c|c|c|}
\hline & $\chi^{2}$ & $\mathrm{gl}$ & CFI & GFI & RMSEA & $\chi^{2} \operatorname{dif}$ & $\Delta \mathrm{CFI}$ & $\Delta \mathrm{GFI}$ & $\triangle \mathrm{RMSEA}$ \\
\hline Modelo 1 & $183.84^{*}$ & 118 & 0.90 & 0.85 & 0.07 & & & & \\
\hline Modelo 2 & $72.71^{*}$ & 53 & 0.95 & 0.91 & 0.06 & & & & \\
\hline Dif. M1 y M2 & & & & & & $111.13 *$ & 0.05 & 0.06 & 0.01 \\
\hline Modelo 3 & $186.88^{*}$ & 119 & 0.89 & 0.84 & 0.07 & & & & \\
\hline Modelo 2 & $72.71^{*}$ & 53 & 0.95 & 0.91 & 0.06 & & & & \\
\hline Dif. M3 y M2 & & & & & & $114.17^{*}$ & 0.06 & 0.07 & 0.01 \\
\hline Modelo 1 & $183.84^{*}$ & 118 & 0.90 & 0.85 & 0.07 & & & & \\
\hline Modelo 3 & $186.88^{*}$ & 119 & 0.89 & 0.84 & 0.07 & & & & \\
\hline Dif. M1 y M3 & & & & & & 3.04 & 0.01 & 0.01 & 0.00 \\
\hline
\end{tabular}

Nota. $* p<.01$

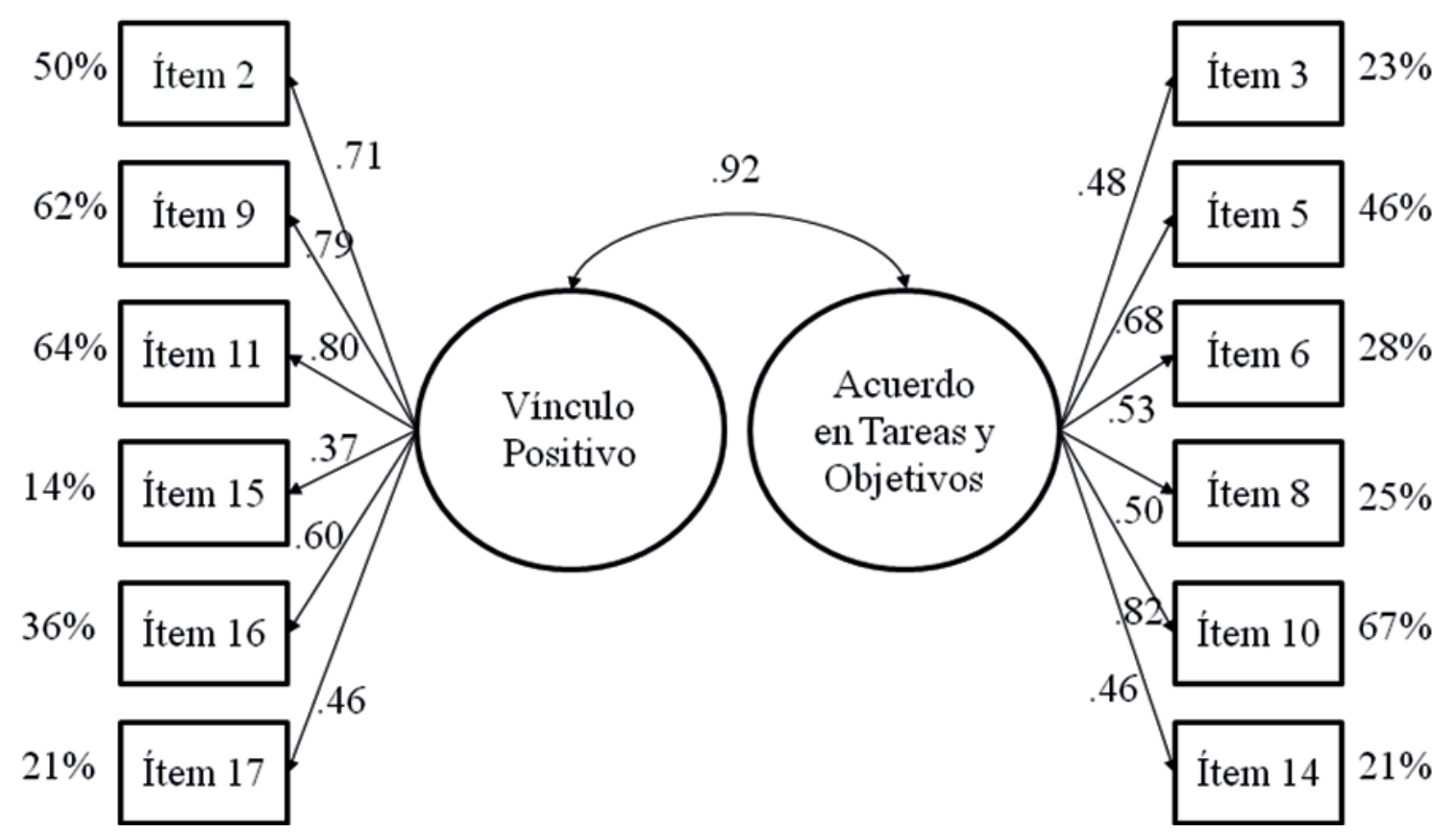

Figura 1. Análisis factorial confirmatorio del Modelo 2 con coeficientes de regresión estandarizados y proporción de varianza explicada para cada item.

Finalmente se analizaron si existían diferencias en los niveles de ambas dimensiones de la alianza considerando el género y el tipo de terapeuta. Utilizando el procedimiento de ANOVA factorial $2 \times 2$ se determinó si existían diferencias según el género, el tipo de terapeuta o un efecto interactivo entre ambas variables. No se observaron efectos interactivos o principales de género $(F$ $=43.92 ; g l=1 ; p=.09)$ o terapeuta $(F=.68 ; g l=1 ; p$ $=.79)$ al considerar la "Alianza Positiva". Al considerar el "Acuerdo en los Objetivos y Tareas" no se observaron efectos principales significativos según el género $(F=$ $1.47 ; g l=1 ; p=.22)$ o el tipo de terapeuta $(F=.51 ; g l$ $=1 ; p=.47)$. No obstante al inspeccionar el gráfico se observó un efecto interactivo, tal como se presenta en la figura 2 los hombres tienden a presentar mayor acuerdo cuando el terapeuta es un psicólogo, mientras que las mujeres tienden a presentar mayor acuerdo cuando el terapeuta es un psiquiatra. 


\section{Discusión}

Tal como sugieren Norcross y Lambert (2005), el movimiento de Psicología Basada en la Evidencia resulta incompleto si se deja de lado la importancia de la relación terapéutica y de los intercambios interpersonales. Tal como señalan estos autores este factor realiza aportes sustanciales a los resultados terapéuticos. Si bien tanto el terapeuta como el paciente determinan la calidad de la relación, los pacientes atribuyen el éxito del tratamiento directamente al terapeuta y al grado en que el terapeuta le ha provisto de empatía, apoyo y respeto en el proceso terapéutico. De acuerdo a lo reportado por estos autores, la relación terapéutica explica al menos el 10\% de la varianza de los resultados terapéuticos, añadiéndose un $8 \%$ de los efectos provistos por el terapeuta, y entre un $5 \%$ y $8 \%$ del método utilizado. Más aún algunos autores llegan a proponer que la alianza terapéutica es el factor más importante de la psicoterapia (Opazo, 2001; Orlinsky, Ronnestad y Willutzki, 2004; Santibáñez, Román y Vinet, 2009).

En función de lo anterior la División de Psicoterapia de la APA recomienda animar a los psicoterapeutas para (Norcross, 2001): 1) crear y cultivar la alianza terapéutica; 2) adaptar la relación terapéutica a las características del paciente; y 3) monitorear las respuestas del paciente frente a la relación terapéutica y al tratamiento. Sin embargo difícilmente pueden llevarse a cabo estas acciones si no se cuenta con instrumentos adecuados y debidamente adaptados al contexto local que permitan evaluar la alianza terapéutica.
El presente trabajo tuvo por objetivo principal efectuar una adaptación del Cuestionario de Alianza de Ayuda Revisado Versión Paciente (HAq-II-P) de Luborsky et al. (1996). Si bien los resultados psicométricos son satisfactorios la versión adaptada difiere considerablemente de la versión original de Luborsky et al. (1996). En primera instancia se reduce la cantidad de ítems dado que la escala original contaba con 17 reactivos mientras que la versión adaptada cuenta con 12 . Cabe señalar que los 5 ítems restantes fueron eliminados en función de un criterio más teórico que psicométricos dado que se consideró que éstos no reflejaban adecuadamente las dimensiones examinadas de alianza terapéutica.

La principal diferencia con la escala original se observa en la estructura factorial, dado que la versión adaptada cuenta con dos dimensiones de alianza terapéutica, la alianza tipo uno más relacionada con el vínculo positivo entre terapeuta y paciente, y la alianza tipo dos que involucra el acuerdo con las tareas y los objetivos. El modelo propuesto posee mayor coherencia teórica y un ajuste significativamente superior que el modelo original de Luborsky et al. (1996) de alianza positiva y negativa. En este punto cabe señalar que el segundo factor identificado en el estudio original (alianza terapéutica negativa) estuvo constituido solamente por 4 ítems y explicaba únicamente un 10,6\% de la varianza. En consecuencia este factor resulta débil y la estructura factorial propuesta constituye una solución poco robusta. Una revisión de la teoría en la cual se basa este cuestionario no ofrece aclaraciones definitivas acerca de una estructura factorial esperable. Este aspecto no

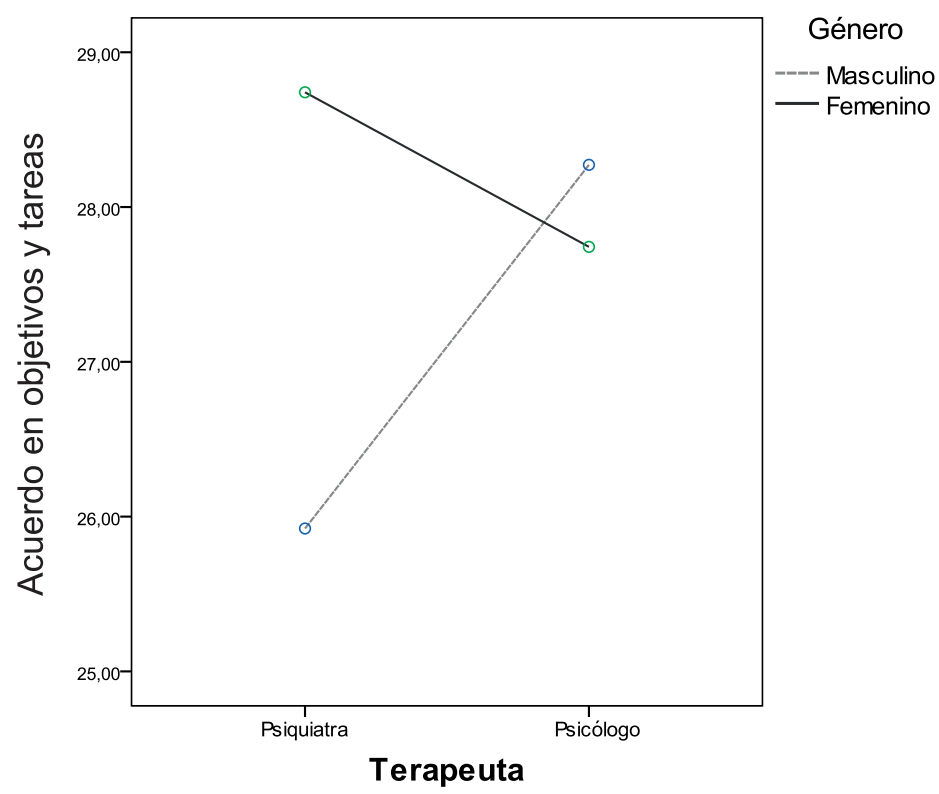

Figura 2. Efecto interactivo entre tipo de terapeuta y género del paciente sobre los niveles de acuerdo en los objetivos y tareas. 
es menor dado que el cuestionario HAq-II fue creado a partir de la revisión del Cuestionario de Alianza de Ayuda (Helping Alliance Questionnaire-Haq) con el propósito de representar mejor la alianza terapéutica según las teorías de Luborsky y Bordin (Luborsky et al., 1996). Si bien el HAq-II posee propiedades psicométricas satisfactorias en términos de fiabilidad y validez, se basa en un modelo teórico inadecuadamente definido dado que éste no se corresponde con ninguno de los modelos anteriormente propuestos por Luborsky (1994) y Bordin (1976). En contraposición el modelo finalmente adoptado en la versión adaptada del HAq-II integra ambos modelos logrando así una medición conceptualmente más significativa y de mayor coherencia teórica.

En relación a la consistencia interna de las escalas no se obtuvieron valores tan elevados como los reportados en el estudio original (coeficientes $\alpha$ entre .90 y .93). No obstante los niveles de homogeneidad de cada escala son buenos atendiendo a los criterios propuestos por Oviedo y Campos-Arias (2005).

En cuanto a los estudios de contrastación de grupos según el género de los pacientes y el tipo de terapeuta se pudo observar un efecto de interacción. Tal como reflejan los resultados los hombres tienden a presentar mayor acuerdo cuando el terapeuta es un psicólogo, mientras que las mujeres tienden a presentar mayor acuerdo cuando el terapeuta es un psiquiatra. En función de estos resultados podría suponerse que la modalidad terapéutica del psiquiatra puede favorecer el acuerdo con los objetivos y las tareas. No obstante, se ha encontrado que la alianza está facilitada cuando el paciente y terapeuta son del mismo género (Luborsky, 1994; Wintersteen, Mensinger y Diamond, 2005) y, por lo tanto, podría ser que en este estudio el fenómeno de mayores puntuaciones de alianza en mujeres con psiquiatras y de hombres con psicólogos se debe a una mayor ocurrencia de diadas paciente-terapeuta del sexo femenino (en el caso de los psiquiatras) y diadas del sexo masculino (en el caso de los psicólogos).

\section{Limitaciones y futuras líneas de trabajo}

Este estudio cuenta con varias limitaciones. En cuanto a la confiabilidad, se carece de estudios acerca de la estabilidad ya que no se realizaron estudios test-retest. Otra restricción es que sería conveniente replicar los análisis con una muestra de mayor tamaño a los fines de garantizar mayor estabilidad en la estructura factorial. En este sentido sería recomendable efectuar estudios de validación cruzada con otras muestras a los fines de examinar si se mantiene la estructura subyacente de dos factores. Otra limitación es que no se llevaron a cabo estudios acerca de la validez convergente discriminante del cuestionario, o bien estudios de evidencia de validez con variables externas como por ejemplo, la adherencia al tratamiento o la eficacia del mismo, por ejemplo.

Por otro lado, este es el primer cuestionario adaptado al ámbito local que permite que el paciente haga una evaluación de la alianza que se ha establecido con su terapeuta $\mathrm{y}$, a pesar de sus limitaciones, este trabajo abre la vía para nuevos líneas de desarrollo en nuestro país tanto a nivel teórico como práctico. El cuestionario puede ser utilizado localmente en la investigación de temáticas actuales del campo de estudio de la alianza como, por ejemplo, las rupturas en la alianza y la reparación de alianzas. También puede ser utilizado en estudios para confirmar, modificar $\mathrm{y} / \mathrm{o}$ profundizar la teoría ya establecida acerca de los factores que influyen en la alianza y la relación entre la alianza y los resultados terapéuticos.

\section{Implicancias prácticas}

Safran y Muran (2003), apoyan la idea de que es necesario que haya una negociación entre terapeuta y paciente sobre las tareas y los objetivos a trabajar en la terapia, y entienden que estos procesos constituyen un punto de mucha importancia para el establecimiento de la alianza. De esta manera se toman distancia de concepciones tradicionales que consideraron que la alianza es responsabilidad únicamente del terapeuta, quien tenía que lograr que el paciente se identificase con él y adoptase sus opiniones sobre las tareas y los objetivos a seguir en la terapia. Al contrario, estos autores consideran que la alianza es una construcción conjunta entre ambos paciente y terapeuta, en la cual se incluyen las ideas, expectativas y las construcciones que ambos van desarrollando respecto del trabajo terapéutico.

A nivel práctico, este cuestionario puede ser utilizado por psicólogos clínicos locales para evaluar las alianzas que se han establecido con los pacientes, permitiendo que se identifiquen debilidades y que se realicen ajustes en su labor clínico para fortalecer la alianza y así fomentar resultados terapéuticos más favorables. Este cuestionario también puede ser utilizado en programas de entrenamiento de psicólogos, como una manera de ayudar a que se desarrollen en los futuros y actuales psicólogos conductas facilitadoras de una buena alianza, lo cual promovería la eficacia del trabajo psicoterapéutico localmente. 


\section{Referencias}

APA Presidential Task Force on Evidence-Based Practice (2006). Evidence-based practice in psychology. American Psychologist, 61, 271-285.

Beck, A., Rush, A., Shaw, B., y Emery, G. (2005). Terapia Cognitiva de la Depresión (16 ${ }^{\mathrm{a}}$ ed.). Bilbao: Desclée de Brower

Bordin, E. S. (1976). The Generalizability of the Psychoanalytic Concept of the Working Alliance. Psychotherapy: Theory, Research and Practice, $16,252-260$.

Byrne, B. (2001). Structural Equation Modelling with Amos. London: Erlbaum Asociados

Coffman, M. J. (2008). Translation of a Diabetes Self-Effycacy Instrument: Assuring Content and Semantic Equivalence. The Journal of Theory Construction and Testing, 12, 58-62.

Corbella, S., y Botella, L. (2003). La Alianza Terapéutica: Historia, Investigación y Evaluación. Anales de Psicología, 19, 205-221.

Crits-Christoph, P., Gladis, M., y Connolly, M. B. (2002). Outcome Measurement in Patients Receiving Psychosocial Treatments. En W. W. IsHak, T. Burt y L. I. Sederer (Eds), Outcome Measurement in Psychiatry: A Critical Review. (pp. 121-138). Washington, DC: American Psychiatric Publishing

Fenton, L. R., Cecero, J. J., Nich, C., Frankforter, T. L., y Carroll, K. M. (2001). Perspective is Everything: the Predictive Validity of Six Working Alliance Instruments. Journal of Psychotherapy Practice and Research, 10, 262-268.

Fernández, A. (2008). Adaptación de Tests a Otras Culturas. En S. Tornimbeni, E. Pérez y F. Olaz, Introducción a la Psicometría (pp. 191-205). Buenos Aires: Paidos.

Gaston, L. (1991). Reliability and Criterion-Related Validity of the California Psychotherapy Alliance Scales- Patient Version. Psychological Assessment: A Journal of Consulting and Clinical Psychology, 3, 68-74.

Gelso, C. J., y Carter, J. A. (1994). Components of the Psychotherapy Relationship: Their interaction and Unfolding During Treatment. Journal of Counselling Psychology, 41, 296-306.

George, D., y Mallery, P. (2010). SPSS for Windows Step by Step: A Simple Guide and Reference, 17.0 update $\left(10^{\mathrm{a}} \mathrm{ed}\right.$.) Boston: Pearson

Gómez, B. (2010). La Relación Terapéutica en Terapia Cognitiva. Revista de la Asociación de Psicoterapia de la Republica Argentina, 1, (online).

Goodheart, C. D., Kazdin, A. E., y Sternberg, R. J. (Eds.). (2006). Evidencebased Psychotherapy: Where Practice and Research Meet. Washington DC: American Psychological Association.

Hambleton, R. K., y Patsula, L. (1999). Increasing the Validity of Adapted Tests: Myths to be Avoided and Guidelines for Improving Test Adaption Practises. Applied Testing Technology, 1, 1-16.

Horvath, A. O., y Greenberg, L. S. (1986). Development of the Working Alliance Inventory. En L. S. Greenberg y W. M. Pinsoff (Eds.), The Psychotherapeutic Process: A Research Handbook (pp. 529-556). New York: Guilford.

Horvath, A. O., y Greenberg, L. S. (Eds.). (1994). The Working Alliance: Theory Research and Practice. New York: Wiley y Sons.

Horvath, A. O., y Symonds, B. D. (1991) Relationship Between Working Alliance and Outcome in Psychotherapy: A meta Analysis. Journal of Consulting and Clinical Psychology, 38, 139-149.

Hu, L. T., y Bentler, P. (1995). Evaluating Model Fit. En R. H. Hoyle (Ed.), Structural Equation Modeling. Concepts, Issues and Applications (pp. 76-99). London: Sage.

Le Bloc'h, Y., de Roten, Y., Drapeau, M., y Despland, J. N. (2006). New, But Improved? Comparison Between First and Revised Version of the Helping Alliance Questionnaire. Schweizer Arch Fur Neurologie Und Psychiatrie, 157, 23-28.
León, O. G., y Montero, I. (2003). Métodos de Investigación en Psicología y Educación ( $3^{\mathrm{a}}$ Ed.). México: McGraw Hill.

Luborsky, L. (1994). Therapeutic Alliances as Predictors of Psychotherapy Outcomes: Factors Explaining the Predictive Success. En A. O. Horvath y L. S. Greenberg (Eds.), The Working Alliance Theory Research and Practice (pp 38-50). New York: John Wiley and Sons.

Luborsky, L., Barber, J. P., Siqueland, L., Johnson, S., Najavits, L. M., ... Frank, A. (1996). The Revised Helping Alliance Questionnaire (HAqII) Psychometric Properties. Journal of Psychotherapy Practice and Research, 5, 260-271.

Marmar, C. R., Gaston, L., Gallagher, D., y Thomson, L. W. (1989). Alliance and Outcome in Late Life Depression. The Journal of Nervous and Mental Disease, 177, 464-472.

Martin, D. J., Garske, J. P., y Davis, M. K. (2000). Relation of the Therapeutic Alliance With Outcome and Other Variables: A Meta-Analytic Review. Journal of Consulting and Clinical Psychology, 68, 438-450.

Montero, I., y León, O. (2007). A Guide for Naming Research Studies in Psychology. International Journal of Clinical and Health Psychology, 7, 847-862.

Muñiz, J., y Hambleton, R. K. (1996). Directrices Para la Traducción y Adaptación de los Tests. Papeles Del Psicólogo, 66, 63-70.

Norcross, J. C. (2001). Purposes, process, and products of the task force on empirically supported therapy relationships. Psychotherapy: Theory, Research, Practice, Training, 38, 345-356.

Norcross, J. C., y Lambert, M. J. (2005). What should be validated? The therapy relationship. En J. C. Norcross, L. E. Beutler y R. F. Levant (Eds.). Evidence-based practices in mental health (208-218). Washington: American Psychological Association.

Opazo, R. (2001). Psicoterapia Integrativa: Delimitación Clínica. Santiago de Chile: Ediciones ICPSI.

Orlinsky, D., Ronnestad, M., y Willutzki, U. (2004). Fifty years of psychotherapy process-outcome research: Continuity and change. En M. Lambert (Ed.), Bergin and Garfield's handbook of psychotherapy and behavior change (pp. 307-393). Nueva York: Wiley.

Oviedo, H. C., y Campo-Arias, A. (2005). Aproximación al Uso del Coeficiente Alfa de Cronbach. Revista Colombiana de Psiquiatría, $34,572-580$.

Rodríguez-Ayán, M. N., y Ruiz, M. A. (2008). Atenuación de la Asimetría y de la Curtosis de las Puntuaciones Observadas Mediante Transformaciones de Variables: Incidencia Sobre la Estructura Factorial. Psicológica, 29, 205-227.

Safran, J. D., y Muran, J. C. (2003). Negotiating the Therapeutic Alliance: A Relational Treatment Guide. New York: Guilford Press

Santibáñez, P. M., Román, M. F., y Vinet, E. V. (2009). Efectividad de la psicoterapia y su relación con la alianza terapéutica. Interdisciplinaria, 26, 267-287.

Soto, C. M., y Segovia, J. L. (2009). Intervalos de Confianza Asimétricos para el Índice de Validez de Contenido: Un Programa Visual Basic para la V de Aiken. Anales de Psicología, 25, 169-171.

Stevens, K., y Méndez, D. (2007). Nuevas Herramientas para la Psicología Clínica: Las Terapias Basadas en la Evidencia. Cuadernos de Neuropsicología, 1, 265-272

Summers, R. F., y Barber, J. P. (2003). Therapeutic Alliance as a Measurbale Psychotherapy Skill. Academic Psychiatry, 27, 160-165.

Wintersteen, M., Mesinger, J., y Diamond, G. (2005). Do Gender and Racial Differences Between Patient and Therapist Affect Therapeutic Alliance and Treatment Retention in Adolescents? Professional Psychology: Research and Practice, 36, 400-408. 